\title{
Frequency of Hawking radiation of black holes
}

\author{
Dipo Mahto ${ }^{1}$, Brajesh Kumar $\mathrm{Jha}^{2}$, Krishna Murari Singh ${ }^{1}$, Kamala Parhi ${ }^{3}$ \\ ${ }^{1}$ Dept. of Physics, Marwari College, T.M.B.U. Bhagalpur-812007, India \\ ${ }^{2}$ Deptartment of Physics, L.N.M.U. Darbhanga, India \\ ${ }^{3}$ Dept. of Mathematics, Marwari College, T.M.B.U. Bhagalpur-812007, India
}

\section{Email address:}

dipomahto@hotmail.com(D. Mahto),brajeshjha111@gmail.com(B. K. Jha), kmsinghphy@gmail.com(K. M. Singh), parhikamala@gmail.com (K. Parhi)

\section{To cite this article:}

Dipo Mahto, Brajesh Kumar Jha, Krishna Murari Singh, Kamala Parhi. Frequency of Hawking Radiation of Black Holes. International Journal of Astrophysics and Space Science. Vol. 1, No. 4, 2013, pp. 45-51. doi: 10.11648/j.ijass.20130104.15

\begin{abstract}
In the present research work, we calculate the frequencies of Hawking radiations emitted from different test black holes existing in X-ray binaries (XRBs) and active galactic nuclei (AGN) by utilizing the proposed formula for the frequency of Hawking radiation $f=\frac{8.037 \times 10^{33} \mathrm{~kg}}{M} \mathrm{~Hz}$ and show that these frequencies of Hawking radiations may be the components of electromagnetic spectrum and gravitational waves. We also extend this work to convert the frequency of Hawking radiation in terms of the mass of the sun $\left(M_{\odot}\right)$ and then of Chandrasekhar limit $\left(M_{c h}\right)$, which is the largest unit of mass.
\end{abstract}

Keywords: Electromagnetic Spectrum, Hawking Radiation, XRBs and AGN

\section{Introduction}

A black hole is a solution of Einstein's gravitational field equations in the absence of matter that describes the space time around a gravitationally collapsed star. Its gravitational pull is so strong that even light cannot escape from it (Dabholkar 2005, Mahto et al. 2012\&2013). The space-time having a black hole in it, first, has a singularity, and second, has a horizon preventing an external observer from seeing it. The singularity in GR is radically different from field theory singularities because it is a property not of some field but of the space-time itself. The topology of space-time is changed when it acquires a black hole (Soloviev, 2005).

In classical theory black holes can only absorb and not emit particles. However, it is shown that quantum mechanical effects cause black holes to create and emit particles as if they were hot bodies with temperature $\frac{h \kappa}{2 \pi k} \approx 10^{-6}\left(\frac{M_{\odot}}{M}\right) \kappa$, where $\kappa$ is surface gravity of black holes and $\mathrm{k}$ is the Boltzmann constant. This thermal emission leads to show a decrease in the mass of the black hole and to its eventual disappearance: any primordial black hole of mass less than about $10^{15}$ gram would have evaporated by now (Hawking, 1975).

Hawking's work followed his visit to Moscow, where Soviet scientists Yakov Zeldovich and Alexander
Starobinsky showed him that according to the quantum mechanical uncertainty principle, rotating black holes should create and emit particles (Hawking, 1988).

Before Hawking, in 1969, Leonard Parker obtained an expression for the average particle density as a function of time, and showed that particles creation is in pairs. The canonical creation and annihilation operators corresponding to physical particles during the expansion of the universe are specified and argued that in the present predominantly dust-filled universe, only massless particles of zero spin might possibly be produced in significant amounts by the present expansion and also showed that massless particles of arbitrary non-zero spin, such as photons or gravitons, are not created by the expansion regardless of its form (Parker, 1969).

Hawking radiation arises for any test field on any Lorentzian geometry containing an event horizon regardless of whether or not the Lorentzian geometry satisfies the dynamical Einstein equations of general relativity (Matt., 1997).

The outgoing wave packet is composed of wave vectors around $\mathrm{K}+\mathrm{s}$ (and has positive Killing frequency) and arises from a pair of packets composed of wave vectors around $\mathrm{K}+$ and $\mathrm{K}$ - respectively which have positive and negative free-fall frequency respectively (Corely and Jacobson 1998).

Steven Corely and Ted Jacobson found that there are two qualitatively different types of particle production in this 
model: a thermal Hawking flux generated by "mode conversion" at the black hole horizon, and a non-thermal spectrum generated via scattering off the background into negative free-fall frequency modes. This second process has nothing to do with black holes and does not occur for the ordinary wave equation because such modes do not propagate outside the horizon with positive Killing frequency (Corely and Jacobson 1996).

Compact binaries (two neutron stars, two black holes, one $\mathrm{BH}$ and one NS, binary stars, rotating neutron stars, neutron star instabilities, super nova, super massive black holes and stochastic background) are the sources emitting gravitational waves (Daniel Sigg, 1998). Gravitational wave detection effects focus on four frequency bands (Curt and Thorne, 2002) (i) The extremely low frequency band $\left(10^{-15}\right.$ to $10^{-18} \mathrm{~Hz}$ ).

(ii) The very low frequency band $\left(10^{-7}\right.$ to $\left.10^{-9} \mathrm{~Hz}\right)$.

(iii) The low frequency band $\left(10^{-4}\right.$ to $\left.1 \mathrm{~Hz}\right)$.

(iv) The high frequency band ( 1 to $10^{4} \mathrm{~Hz}$ ).

In the present research paper, we have calculated the frequency of Hawking radiation of different types of the test black holes existing in X-ray binaries (XRBs) and active galactic nuclei (AGN) emitted by black holes and we have also tried to show that frequencies of Hawking radiations emitted by black holes may be the components of electromagnetic spectrum. This work is further extended to convert the frequency of Hawking radiation in terms of mass of the sun and then in Chandrasekhar limit $\left(M_{c h}\right)$.

\section{Theoretical Discussion}

Classically, the black holes are perfect absorbers and do not emit anything; their temperature is absolute zero. However, in quantum theory black holes emit Hawking radiation with a perfect thermal spectrum. This allows a consistent interpretation of the laws of black hole mechanics as physically corresponding to the ordinary laws of thermodynamics (Wald 2001).

In quantum physics, the empty space is not empty at all. In it there are always particles flashing into existence and disappear again. They always come in pairs; one particle and one anti-particle, like an electron and a positron, or a photon and another photon with opposite spin and impulse. These particles are called virtual particles. They only exist for a very short time given by (Hawking radiation, htt://library.thinkquest.org/c007571/English/printcore.htm, 2011)

$$
\Delta t=\frac{1}{8 \pi f}
$$

where $f$ is the frequency of radiation. If one virtual particle falls into the black hole and the other escapes, it escapes as Hawking radiation from black hole. This radiation shows the same allocation as black body radiation. This assumes that black holes do have a temperature too.

Once, we accept that black holes can radiate, then it is not hard to estimate the wavelength of the radiation that they emit. The only length-scale in the problem is the size of the horizon. A photon with a wavelength $(\lambda)$ equal to the radius of the black hole has energy equal to

$$
E=h v
$$

The energy of a pair of virtual photons will be given by the following equation

$$
E=2 h v
$$

The energy of a photon of Hawking radiation is given by the following equation (Hawking radiation, htt://ibrary.thinkquest.org/c007571/English/printcore.htm, 2011)

$$
E=\frac{h c^{3}}{16 \pi G M}
$$

From equation (2) and (4), we have

$$
v=\frac{c^{3}}{16 \pi G M}
$$

All the terms like gravitational constant (G), Planck constant (h) and velocity of light(c) on the right hand side of the equation (5) are constant except mass (M) of the black hole. These constants have vital role discussed as:

The three fundamental constants of nature - the speed of light (c), Planck's constant (h) and Newton's gravitational constant $(\mathrm{G})$ are present in the $\mathrm{eq}^{\mathrm{n}}(5)$. Planck's constant (h) governs the law of quantum world. The speed of light (c) is the cornerstone of the special theory of relativity. The fact that light is an electromagnetic wave travelling at the speed of light (c) is very important consequences of Maxwell's equations for electromagnetic field. In general relativity, Newton's gravitational constant G has an entirely new meaning. For Newton, $\mathrm{G}$ is the constant of proportionality that appears in inverse square law of gravitation, while for Einstein; $\mathrm{G}$ is a constant that determines the degree to which a given distribution of matter warps space and time (Dabholkar, 2005).

The equation (5) can be written as

$$
v \propto \frac{1}{M}
$$

If $\lambda$ be the wavelength of Hawking radiation emitted from the black hole, then we have

$$
\lambda \propto M
$$

The relation (6) shows that the frequency of Hawking radiation emitted by the black holes is inversely proportional to the mass of the black hole, whereas from relation (7) it is clear that the wavelength of Hawking radiation emitted by the black hole is directly proportional to the mass of the black holes. This means that the heavier black hole will emit the Hawking radiation of lower frequency or longer 
wavelength and vice-versa.

Putting the values of $h, c, G$ and $\pi$ in equation (5), we have

$$
\begin{gathered}
v=\frac{8.037 \times 10^{33} \mathrm{~kg}}{M} \mathrm{~Hz} \\
\text { Or } v=\frac{8.037 \times 10^{33} \times 1.99 \times 10^{30} \mathrm{~kg}}{M \times 1.99 \times 10^{30}} \mathrm{~Hz}
\end{gathered}
$$

The mass of the sun $\left(M_{\odot}\right)$ is given by (Narayan, $2005 \&$ Mahto et al. 2011)

$$
M_{\odot}=1.99 \times 10^{30} \mathrm{~kg}
$$

Putting the value of eq ${ }^{\mathrm{n}}(10)$ in $\mathrm{eq}^{\mathrm{n}}(9)$, we have

$$
\begin{gathered}
v=\frac{4.0386 \times 10^{3} \times M_{\odot}}{M} H z \\
v=4.0386 \times 10^{3}\left(\frac{M_{\odot}}{M}\right) H z \\
v=4.0386\left(\frac{M_{\odot}}{M}\right) K H z \\
v=\frac{4.0386}{1.4}\left(\frac{1.4 M_{\odot}}{M}\right) K H z \\
v=2.8847\left(\frac{M c h}{M}\right) K H z
\end{gathered}
$$

where $M c h=1.4 M_{\odot}$ is called Chandrasekhar limit which is itself the largest unit of mass used for the measurement of astronomical stellar bodies.

\section{Data in Support of Mass of Black Holes in XRBs and AGN}

There are two categories of black holes classified on the basis of their masses clearly very distinct from each other, with very different masses $\mathrm{M} \sim 5-20 \mathrm{M}_{\odot}$ for stellar-mass black holes in X-ray binaries and $\mathrm{M} \sim 10^{6}-10^{9.5} \mathrm{M}_{\odot}$ for super massive black holes in Galactic nuclei. Masses in the range $10^{6} M_{\odot} t o 3 \times 10^{9.5} M_{\odot}$ have been estimated by this means in about 20 galaxies (Narayan 2005).
The most viable scenario for modeling of active galactic nuclei includes a super massive black hole with the mass $10^{6}-10^{9} M_{\odot}$ accreting the galaxian matter from its vicinity (Madejski, 2003).

At the distance of the Virgo cluster, $15 \mathrm{Mpc}$, the sphere of influence of a $\sim 3 \times 10^{7} M_{\odot}$ super-massive black holes would shrink to a projected radius of $0 " .07$, not only well beyond the reach of any ground based telescope, beyond even HST capabilities (Ferrarese \& Ford, 2005).

Assuming an isotropic, spherically symmetric system, Sargent et al. detected a central dark mass $\sim 5 \times 10^{9} M_{\odot}$ within the inner 110pc of M87 (Sargent et al., 1978).

Assuming the disk is Keplerian, Greenhill and Gwinn estimated the mass enclosed within $0.65 \mathrm{pc}$ to be $\sim 1.5 \times 10^{7} M_{\odot}$ (Ferrarese, \& Ford, 2005).

In NGC 4041, acquiescent Shc spiral, Marconi et al. (2003) remark that the systematic blue shift of the disk relative to systemic velocity might be evidence that the disk is kinematically decoupled. They conclude that only an upper limit, of $2 \times 10^{7} M_{\odot}$, can be put on the central mass. Cappellari et al. (2002) conclude that non-gravitational motions might indeed be present in the case of IC 1459, for which the ionized gas shows no indication of rotation in the inner 1 " IC 1459 is the only galaxy for which a super massive black hole mass estimate exists based both on gas and stellar kinematics. Three-integral models applied to the stellar kinematics produce, $M_{\bullet}=(2.6 \pm 1.1) \times 10^{9} M_{\odot}$, while the gas kinematics produces estimates between a few $\times 10^{8}$ and $10^{9} M_{\odot}$, depending on the assumptions made regarding nature of the gas velocity dispersion(Ferrarese \& Ford, 2005).

Masses of "central dark object" have been estimated in about forty cases, using stellar dynamics, emission lines of orbiting gas and, most accurately, using water masers. They range from $\sim 2 \times 10^{6} M_{\odot} t o \sim 3 \times 10^{9} M_{\odot}$ and, in many cases, the compactness is sufficient to rule out star clusters with confidence (Blandford, 1999).

Most detected super massive black holes are in the $10^{8} \leq M_{\bullet} \leq 10^{9} M_{\odot}$ range, there are no detections below $10^{6} M_{\odot}$ (the "building block" range) or above $10^{10} M_{\odot}$ (the brightest quasar range), and even the $10^{6} \leq M_{.} \leq 10^{7} M_{\odot}$ range is very poorly sampled (Ferrarese \& Ford, 2005). 


\section{Table}

\subsection{Table 1}

\begin{tabular}{|c|c|c|c|}
\hline \multicolumn{4}{|c|}{ Wavelength and frequency of Hawking radiation in XRBs. } \\
\hline Sl. No & $\begin{array}{l}\text { Mass of BHs (M) } \\
M_{\odot}=1.99 \times 10^{30} \mathrm{~kg}\end{array}$ & Frequency of Hawking radiation $(\mathrm{Hz})$ & $\begin{array}{l}\text { Wavelength of Hawking radiation ( } \lambda \text { in } \\
\text { metre). }\end{array}$ \\
\hline 1 & $5 \mathrm{M}_{\square}$ & $8.037 \times 10^{2}$ & $3.714 \times 10^{5}$ \\
\hline 2 & $6 \mathrm{M}_{\Xi}$ & $6.731 \times 10^{2}$ & $4.456 \times 10^{5}$ \\
\hline 3 & $7 \mathrm{M}_{\Xi}$ & $5.769 \times 10^{2}$ & $5.119 \times 10^{5}$ \\
\hline 4 & $8 \mathrm{M}_{\square}$ & $5.048 \times 10^{2}$ & $5.942 \times 10^{5}$ \\
\hline 5 & $9 \mathrm{M}_{\varpi}$ & $4.487 \times 10^{2}$ & $6.685 \times 10^{5}$ \\
\hline 6 & $10 \mathrm{M}_{\square}$ & $4.038 \times 10^{2}$ & $7.428 \times 10^{5}$ \\
\hline 7 & $11 \mathrm{M}_{\square}$ & $3.671 \times 10^{2}$ & $8.170 \times 10^{5}$ \\
\hline 8 & $12 \mathrm{M}_{\varpi}$ & $3.365 \times 10^{2}$ & $8.913 \times 10^{5}$ \\
\hline 9 & $13 \mathrm{M}_{\square}$ & $3.106 \times 10^{2}$ & $9.656 \times 10^{5}$ \\
\hline 10 & $14 \mathrm{M}_{\square}$ & $2.884 \times 10^{2}$ & $10.399 \times 10^{5}$ \\
\hline 11 & $15 \mathrm{M}_{\Xi}$ & $2.692 \times 10^{2}$ & $11.142 \times 10^{5}$ \\
\hline 12 & $16 \mathrm{M}_{\square}$ & $2.524 \times 10^{2}$ & $11.885 \times 10^{5}$ \\
\hline 13 & $17 \mathrm{M}_{\varpi}$ & $2.375 \times 10^{2}$ & $12.627 \times 10^{5}$ \\
\hline 14 & $18 \mathrm{M}_{\varpi}$ & $2.243 \times 10^{2}$ & $13.370 \times 10^{5}$ \\
\hline 15 & $19 \mathrm{M}_{\Xi}$ & $2.125 \times 10^{2}$ & $14.113 \times 10^{5}$ \\
\hline 16 & $20 \mathrm{M}_{\varpi}$ & $2.019 \times 10^{2}$ & $14.852 \times 10^{5}$ \\
\hline
\end{tabular}

\subsection{Table 2}

Wavelength and frequency of Hawking radiation in AGN.

\begin{tabular}{cccc} 
S. No. & $\begin{array}{c}\text { Mass of BHs (M) } \\
M_{\odot}=1.99 \times 10^{30} \mathrm{~kg}\end{array}$ & $\begin{array}{c}\text { Frequency of Hawking radiation } \\
(\mathbf{H z})\end{array}$ & $\begin{array}{c}\text { Wavelength of Hawking radiation }(\lambda \text { in } \\
\text { metre) }\end{array}$ \\
\hline 1 & $1 \times 10^{6} \mathrm{M}_{\odot}$ & $4.038 \times 10^{-3}$ & $7.428 \times 10^{10}$ \\
2 & $2 \times 10^{6} \mathrm{M}_{\odot}$ & $2.019 \times 10^{-3}$ & $14.858 \times 10^{10}$ \\
3 & $3 \times 10^{6} \mathrm{M}_{\odot}$ & $1.346 \times 10^{-3}$ & $22.288 \times 10^{10}$ \\
4 & $4 \times 10^{6} \mathrm{M}_{\odot}$ & $1.009 \times 10^{-3}$ & $29.732 \times 10^{10}$ \\
5 & $5 \times 10^{6} \mathrm{M}_{\odot}$ & $0.807 \times 10^{-3}$ & $37.174 \times 10^{10}$ \\
6 & $6 \times 10^{6} \mathrm{M}_{\odot}$ & $0.673 \times 10^{-3}$ & $44.576 \times 10^{10}$ \\
7 & $7 \times 10^{6} \mathrm{M}_{\odot}$ & $0.581 \times 10^{-3}$ & $51.635 \times 10^{10}$ \\
8 & $8 \times 10^{6} \mathrm{M}_{\odot}$ & $0.504 \times 10^{-3}$ & $59.523 \times 10^{10}$ \\
9 & $9 \times 10^{6} \mathrm{M}_{\odot}$ & $0.448 \times 10^{-3}$ & $66.964 \times 10^{10}$ \\
10 & $1 \times 10^{7} \mathrm{M}_{\odot}$ & $4.018 \times 10^{-4}$ & $7.428 \times 10^{11}$ \\
11 & $2 \times 10^{7} \mathrm{M}_{\odot}$ & $2.019 \times 10^{-4}$ & $14.858 \times 10^{11}$ \\
12 & $3 \times 10^{7} \mathrm{M}_{\odot}$ & $1.346 \times 10^{-4}$ & $22.288 \times 10^{11}$ \\
13 & $4 \times 10^{7} \mathrm{M}_{\odot}$ & $1.009 \times 10^{-4}$ & $29.732 \times 10^{11}$ \\
14 & $5 \times 10^{7} \mathrm{M}_{\odot}$ & $0.807 \times 10^{-4}$ & $37.174 \times 10^{11}$ \\
15 & $6 \times 10^{7} \mathrm{M}_{\odot}$ & $0.673 \times 10^{-4}$ & $44.576 \times 10^{11}$ \\
16 & $7 \times 10^{7} \mathrm{M}_{\odot}$ & $0.581 \times 10^{-4}$ & $51.635 \times 10^{11}$ \\
\hline
\end{tabular}




\begin{tabular}{cccc}
\hline \multicolumn{5}{c}{$\begin{array}{c}\text { Wavelength and frequency of Hawking radiation in AGN. } \\
\text { S1. No. }\end{array}$} & $\begin{array}{c}\text { Mass of BHs }(\mathbf{M}) \\
M_{\odot}=1.99 \times 10^{30} \mathrm{~kg}\end{array}$ & $\begin{array}{c}\text { Frequency of Hawking radiation } \\
(\mathbf{H z})\end{array}$ & $\begin{array}{c}\text { Wavelength of Hawking radiation }(\lambda \text { in } \\
\text { metre). }\end{array}$ \\
\hline 17 & $8 \times 10^{7} \mathrm{M}_{\odot}$ & $0.504 \times 10^{-4}$ & $59.523 \times 10^{11}$ \\
18 & $9 \times 10^{7} \mathrm{M}_{\odot}$ & $0.448 \times 10^{-4}$ & $66.964 \times 10^{11}$ \\
19 & $1 \times 10^{8} \mathrm{M}_{\odot}$ & $4.018 \times 10^{-5}$ & $7.466 \times 10^{12}$ \\
20 & $2 \times 10^{8} \mathrm{M}_{\odot}$ & $2.019 \times 10^{-5}$ & $14.858 \times 10^{12}$ \\
21 & $3 \times 10^{8} \mathrm{M}_{\odot}$ & $1.346 \times 10^{-5}$ & $22.288 \times 10^{12}$ \\
22 & $4 \times 10^{8} \mathrm{M}_{\odot}$ & $1.009 \times 10^{-5}$ & $29.732 \times 10^{12}$ \\
23 & $5 \times 10^{8} \mathrm{M}_{\odot}$ & $0.807 \times 10^{-5}$ & $37.174 \times 10^{12}$ \\
24 & $6 \times 10^{8} \mathrm{M}_{\odot}$ & $0.673 \times 10^{-5}$ & $44.576 \times 10^{12}$ \\
25 & $7 \times 10^{8} \mathrm{M}_{\odot}$ & $0.581 \times 10^{-5}$ & $51.635 \times 10^{12}$ \\
26 & $8 \times 10^{8} \mathrm{M}_{\odot}$ & $0.504 \times 10^{-5}$ & $59.523 \times 10^{12}$ \\
27 & $9 \times 10^{8} \mathrm{M}_{\odot}$ & $0.448 \times 10^{-5}$ & $66.964 \times 10^{12}$ \\
28 & $1 \times 10^{9} \mathrm{M}_{\odot}$ & $4.018 \times 10^{-6}$ & $7.466 \times 10^{13}$ \\
29 & $2 \times 10^{9} \mathrm{M}_{\odot}$ & $2.019 \times 10^{-6}$ & $14.858 \times 10^{13}$ \\
30 & $3 \times 10^{9} \mathrm{M}_{\odot}$ & $1.346 \times 10^{-6}$ & $22.288 \times 10^{13}$ \\
31 & $4 \times 10^{9} \mathrm{M}_{\odot}$ & $1.009 \times 10^{-6}$ & $29.732 \times 10^{13}$ \\
32 & $5 \times 10^{9} \mathrm{M}_{\odot}$ & $0.807 \times 10^{-6}$ & $37.174 \times 10^{13}$ \\
\hline
\end{tabular}

\section{Figure}

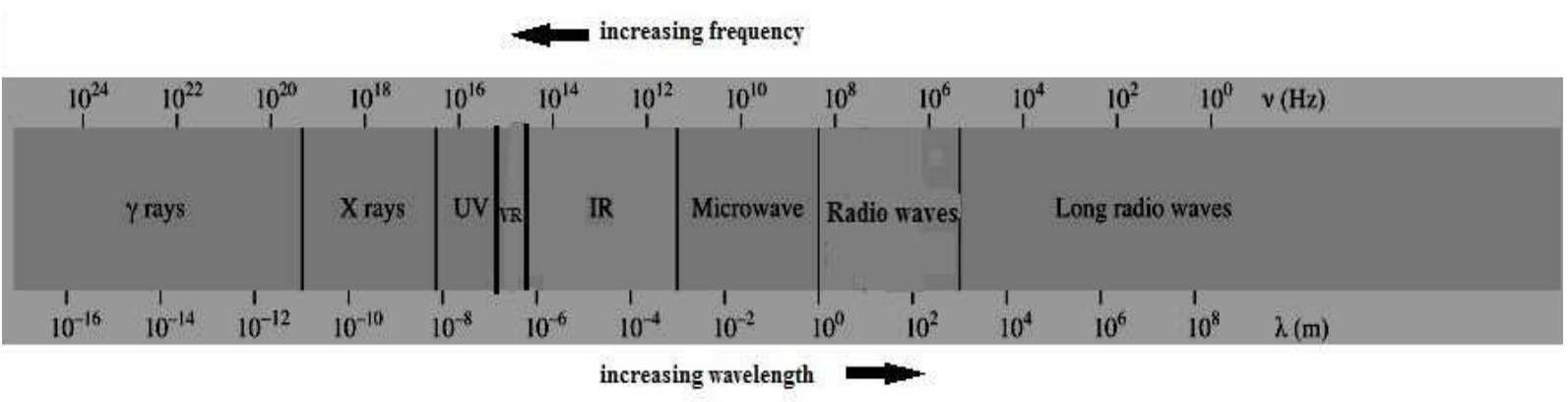

Figure 5.1: Electromagnetic spectrum from Electromagnetic radiation, wikipedia, 2012

\section{Electromagnetic and Gravitational Waves}

Electromagnetic radiation is classified according to the frequency of its wave. The electromagnetic spectrum, in order of increasing frequency and decreasing wavelength, consists of radio waves, microwaves, infrared radiation, visible light, ultraviolet radiation, X-rays and Y-rays. The eyes of various organisms sense a small and somewhat variable window of frequencies of EMR called the visible spectrum. The electromagnetic spectrum is shown in the figure 5.1(Electromagnetic radiation, wikipedia, 2012). According to Einstein's general theory of relativity, when the mass of a system accelerates, the space time surrounding the system undergoes a change in curvature and propagates outward gravitational waves at the speed of light and carries the energy and angular momentum away from the system. Detectable electromagnetic radiation usually originates from the surface of objects, whereas the gravitational waves emanates inside an object. Thus the astronomers expect that the universe as seen in the gravitational waves will look very different from the universe seen in the electromagnetic radiation (http://astro.berky.edu/imran/cosmology1.html).

On the basis of above data for the mass of black holes, we have calculated the frequency and wavelength of Hawking radiation as tabulated in the table 1 for XRBs and in table 2 for AGN. 


\section{Results and Discussion}

While observing table 1, we see that the frequencies / wavelengths of Hawking radiations emitted by black holes in XRBs are few $\times 10^{2} \mathrm{~Hz} /$ few $\times 10^{5}$ metre which are within the range of long radio waves of electromagnetic spectrum and hence these types of radiations may be included in the series of electromagnetic spectrum.

While observing table 2, we see that the frequencies / wavelengths of Hawking radiations emitted by black holes in AGN are lying between $4.018 \times 10^{-3} \mathrm{~Hz}$ to $4.018 \times 10^{-7} \mathrm{~Hz} /$ $7.428 \times 10^{10} \mathrm{~m}$ to $31.174 \times 10^{13} \mathrm{~m}$ which may be placed in the order of long radio waves of electromagnetic spectrum and hence these types of radiations may also be included in the series of electromagnetic spectrum of figure 5.1.

In the present work, the frequencies of Hawking radiations have good agreement with gravitational wave detection efforts given in the reference of Curt \& Thorne (2002). Hence we can say that the Hawking radiations may be gravitational waves. We also see that electromagnetic waves and gravitational waves have quite separate frequencies and wavelengths, but the gravitational waves can be arranged in the order of electromagnetic spectrum.

In this work, we have converted the formula for the frequency of Hawking radiation in terms of mass of the sun $\left(M_{\odot}\right.$ ) also which closely agrees with reference to Denial Sigg (1998) and then in terms of Chandrasekhar limit $\left(M_{c h}\right)$.

From tables 1 and 2, it is clear that the frequencies of Hawking radiations decrease with the increase of mass of different test black holes, while with the increase of mass of different black holes, the wavelength of the Hawking radiation increases.

\section{Conclusion}

In the light of the present work, we can draw the following conclusions:

(1) The Hawking radiations emitted by the black holes may be gravitational waves.

(2) The frequencies of the Hawking radiations emitted by the black holes decrease with the increase of the mass of different test black holes, while the wavelengths of the Hawking radiations increase with the increase of the mass of different test black holes.

(3) The frequencies / wavelengths of Hawking radiations emitted by black holes in XRBs are within the range of long radio waves of electromagnetic spectrum and hence they may be included in the series of electromagnetic spectrum.

(4) The Hawking radiations emitted by the black holes in $\mathrm{XRBs}$ are radio waves, while in AGN are very long radio waves.

(5) The frequencies of the Hawking radiations emitted by the black holes in AGN are below any other components of electromagnetic spectrum and hence they may be placed in the order of long radio waves of electromagnetic spectrum.

(6) The wavelengths of the Hawking radiations emitted by the black holes in AGN are above any other components of electromagnetic spectrum and hence they may be placed in the order of long radio waves of electromagnetic spectrum.

(7) The frequency ranges of the Hawking radiations emitted by the black holes have better agreement with the frequencies of gravitational waves detected by detectors like LIGO, LISA, TAMA etc.

(8) The frequencies / wavelengths of the Hawking radiations emitted by the black holes may be placed in the series of electromagnetic spectrum without any disturbance to it.

(9) The frequency of the Hawking radiation emitted by the black hole may be transformed in terms of the mass of $\operatorname{sun}\left(M_{\odot}\right)$ and then in terms of Chandrasekhar limit $\left(M_{c h}\right)$.

\section{Acknowledgement}

The authors are grateful to the referee for pointing out the errors in the original manuscript and making constructive suggestions. The authors also acknowledge the help of Dr. D. T. K. Dutta, Retired Professor, University Department of English, T. M. B. U., Bhagalpur and Prof. Vijoy Kant Mishra H.O.D. Physics, Marwari College, Bhagalpur in proof reading. The authors are obliged to Dr. Gopi Kant Jha, Former Head \& Prof. of Physics, L.N.M.U Darbhanga and Dr. Neeraj Pant, Associate Professor, Dept. of Mathematics, N.D.A. Khadakwasala, Pune for their inspiration and motivation.

\section{References}

[1] A. Dabholkar: Black hole entropy in string theory-a window in to the quantum structure of gravity. Curr. Sci. 89(12), 25 (2005).

[2] D. Mahto, V. Prakash, B. K. Singh, K. M. Singh: Change in entropy of Non-spinning black holes w.r.t. the radius of event horizon in XRBs, Astrophys SpaceSci. Doi 10.1007/s10509-012-1219-y (2012).

[3] D. Mahto, M.S. Nadeem, U. Prasad, A. Kumar, \& K.M. Singh: Change in entropy of non-spinning black holes w.r,t. the radius of event horizon in AGN. Journal of Modern Physics, 2013, 4, 321-326 doi:10.4236/jmp.2013.43043 Published Online March 2013 (http://www.scirp.org/journal/jmp).

[4] D. Mahto,, R. N. Mehta, N. Pant \& R. K. Sah: Change in Energy of Non-spinning Black Holes w. r. t. the Change in Mass. International Journal of Astronomy and Astrophysics, 2013, 3, 49-56 doi:10.4236/ijaa.2013.31006 Published Online March 2013 (http://www.scirp.org/journal/ijaa).

[5] V.O. Soloviev: Black hole Statistical Physics: Entropy. Institute for High Energy Physics, Protvino, Russia, P54-75, ar Xiv: gr-qc/0507124v1, 29 July 2005.

[6] Daniel Sigg: "Gravitational waves." Publication, LIGO P980007 -00-D, 10/23/98, P-6, 1998.

[7] S. W. Hawking: "Particle Creation by Black Holes." Commun Math. Phys. 43, 199 220, 1977.

[8] S. W. Hawking: A "Brief History of Time". Bantam Books, 
New York (1988). ISBN 0-553-38016-8.

[9] Leonard Parker: "Quantized Fields and Particle Creation in Expanding Universe I.” Physical Review, Vol. 183, issue 5, pp.1050-1068, July 1969. Doi:10.1103/PhysRev.183.1057.

[10] Matt. Visser: "Hawking radiation without black hole entropy.” arXiv: gr-qc/9712016v1, 2 Dec. 1997.

[11] Steven Corley and Ted Jacobson: "Hawking Spectrum and High Frequency Dispersion.” arXiv: hep-th/9601073v1, 15 Jan 1996.

[12] Steven Corley and Ted Jacobson: "Lattice Black Holes." arXiv: hep-th/9709166v2, 26 March, 1998.

[13] Wald, R.M.: The thermodynamics of black holes. Living reviews in relativity. www. livingreviews.org $\backslash$ article $\mid$ volume $2001-6$-wald. (2001)

[14] Curt Cutler \& Kip Thorne: "An overview of gravitational waves sources." arXiv:gr-qc/0204090v1, 30 April, 2002.
[15] R. Narayan: Black Holes In Astrophysics, New Journal Physics, Vol. 7, No. 1, 2005, pp1-31, arXiv: gr-qc/050 6078.

[16] A. Einstein,: On a Stationary System with Spherical Symmetry Consisting of Many Gravitating Masses., Annals of Mathematics, 1939.

[17] Ferrarese, L. \& Ford, H.: "Super massive Black Holes in Galactic Nuclei: Past Present \& Future Research", Space Science Reviews 116, 523-624, 2005, DOI: 10.1007/s11214-005-3947-6.

[18] Ferrarese, L., Ford, H. C. and Jaffe, W.: 1996, APJ 470,444.

[19] Grey Madejski: Black Holes in Active Galactic Nuclei, SLAC-PUB-9702, March 2003.

[20] Blandford, R.D.: "Recent results on Active Galactic Nuclei." Astrophysics and Space Science 261:245-252, 1999. 